\title{
XLVI. Magnetic figures illustrating electrodynamic relations
}

\author{
Silvanus P. Thompson D.Sc. B.A. F.R.A.S.
}

To cite this article: Silvanus P. Thompson D.Sc. B.A. F.R.A.S. (1878) XLVI. Magnetic figures illustrating electrodynamic relations, Philosophical Magazine Series 5, 6:38, 348-353, DOI: $10.1080 / 14786447808639524$

To link to this article: http://dx.doi.org/10.1080/14786447808639524

曲 Published online: 13 May 2009.

Submit your article to this journal

Џ Article views: 5

Q View related articles $₫$ 


\section{[ 348 ]}

XLVI. Magnetic Figures illustrating Electrodynamic Relations. By Silvanus P. Thompson, D.Se., B.A., F.R.A.S., Professor of Experimental Physics in University College, Bristol*

\section{[Plates VI. and VII.]}

TN a preliminary communication to the Physical Society in 1 February of the present year, the author announced a method of studying and illustrating the known laws of the mutual attractions or repulsions of conductors traversed by electric currents. The present paper is a complete statement of the facts obtained in the experimental research which formed the basis of that communication.

While preparing a set of magnetic currents to illustrate the mutual actions of magnet-poles, it occurred to the writer that the mutual attractions and repulsion of currents might be illustrated in a similar manner by the figures formed with iron filings. He was aware ${ }^{2}$ at that time that the lines of force of a strajght conductor carrying a current were a series of concentric circles lying in a plane to which the conductor was normal. The series of figures now published originates, therefore, with the discovery of Faraday that the seat of the mutual actions of currents and of magnets must be sought in the surrounding medium. Since the communication of the preliminary notice, the writer has learned that one or two of the figures had been previously and independently observed by Professor F. Guthrie, but not published. Two others, Nos. 4 and 5 of the present series, are imperfectly given by Faraday in figures 18 and 19 of plate iii. in the third volume of his 'Experimental Researches' (Series Twenty-ninth) $\ddagger$, and without reference to the conclusions to be derived from their forms, which Faraday apparently overlooked $\S$.

The method employed for preserving the figures has been uniform throughout the series. Plates of glass, $3 \frac{1}{2}$ inches long by $3 \frac{1}{4}$ inches broad, were coated with a solution of gum-arabic and gelatine, and were then carefully dried. When the ar-

* Communicated by the Physical Society.

+ See Faraday, 'Experimental Researches in Electricity,' vol. iii. p. 400 , \$ 3239, and plate iii. fig. 17 ; Guthrie, 'Magnetism and Electricity;' p. 254, fig. 225 ; Clerk-Maxwell, 'Electricity and Magnetism,' vol. ii. art. 477.

I And ' Phil. Trans. 1852, p. 137.

The attention of the writer has also been drawn to a statement in the American Journal of Science for 1872, p. 263, by Professor A. M. Mayer, that he has obiained magnetic "spectra "from electric currents in a manner somewhat similar to that now described. The figures have, however, remained unpublished and undescribed, so that the writer has no means of learning how far the substance of the present communication may have been anticipated. 
rangement of magnets or of conducting-wires had been made for the particular case of the experiment, and the plate been laid in a horizontal position, fine filings of wrought iron previously sifted were dusted over the plate through muslin, and the plate was tapped lightly with vertical blows from a piece of thin glass rod. When the filings had arranged themselves, and the plate was still in situ, a gentle current of steam was allowed to play upon the plate, condensing upon the surface of the gum and softening it, and thus allowing the filings to embed themselves where they lay. After the gum had again become hard, the prepared face was covered by a protecting plate of glass, on which in certain cases were drawn the positions of the wires or magnets employed. The figures fixed in this manner are suitable for projection with the lantern upon the screen. They can be readily photographed for transparencies, or for paper prints; specimens of each of these methods of photographic reproduction are exhibited to the Society.

Figure 1 represents the condition of the magnetic field surrounding the current in a straight conducting-wire, which was carried vertically through a hole drilled in the plate. The wire employed throughout the series was a silver one of about .8 millim. in diameter. The battery power employed for this experiment was that of 20 Grove's cells arranged in two series of ten each. In some of the succeeding experiments a less current was found sufficient.

But for the imperfections of the method of experiment, these curves would be perfect circles, and the distances between two successive lines of force would be proportional to the square of the distance from the central point. The equipotential magnetic surfaces, being always normal to the magnetic lines of force, would be represented by a system of radial lines forming equal angles with one another. There appears to be no recognized name for the closed curves traced out by the lines of force around conductors carrying currents. With great diffidence I therefore beg to speak of them as isodynamic lines. They are theoretically disposed about a single straight conductor in a perfectly concentric manner, and at such distances apart as would be defined by the requirement that a parallel conductor, carrying a like current of unit strength, would do unit work in passing from one isodynamic line to the next. The absolute value of an isodynamic line would of course be determined (like magnetic and electrostatic potential) by the work done by a like element of current in passing to any point in that line from an infinite distance. No work is done in moving an element of a parallel current along an isodyna- 
mic line, just as no work is done in moving a magnet-pole along in an equipotential surface. The isodynamic lines occupy, therefore, exactly the same relation to the element of the circuit, as do the equipotential surfaces to a magnet-pole or to an electrified point.

Figure 2 represents the field above a horizontal wire carrying a current, and separated from the filings by the thickness of the glass (about 1.7 millim.). The lines cross the wire at right angles, and are really the projections of a series of such circles as exist in figure 1 .

Figure 3 exhibits the form assumed by the filings when the wire beneath the plate was coiled into a simple loop, a small piece of mica being inserted to prevent contact where it recrossed its path. The lines of the field within the loop run longitudinally; and their projections on the surface are mere points, as the filings show.

In figures 4 and 5 , two wires pass vertically through the plane of the figures, carrying parallel currrents, which in figure 4 are in the same direction, in figure 5 in opposite directions. Ampère's well-known law of the attraction in the former case, and of the repulsion in the latter, is well illustrated by the forms of the magnetic curves. In the former, where the parallel currents attract, the outer isodynamic lines are closed curves embracing both centres, the inner are distorted ovals about each centre-the whole forming a system of lemniscates, as would necessarily be the case, since the attraction at any point in the plate varies inversely as the square of the distance from each current*.

In figure 5, where the parallel currents repel each other, the lines of force due to either current in no case enter or coalesce with those of the other current. They form two series of ovals of a peculiar form, flattened on the sides presented towards the opposing series.

The conception of Faraday, "that the lines of magnetic force tend to shorten themselves, and that they repel each other when placed side by side," has been shown by Clerk-Maxwell, who thus concisely states it, to be perfectly consistent with the theory that explains electromagnetic force as the result of a state of stress in the medium filling the surrounding spacet. Faraday also observes that " unlike magnetic lines, when end on, repel each other, as when similar poles are face to face," and that "like magnetic lines of force," when end on to each other, coalesce. The terms "like" and "unlike," as applied

* See Thomson and Tait, 'Natural Philosophy,' art. 508, vol. i. p. 382.

† Clerk-Maxwell, 'Electricity and Magnetism, vol. ii. art. 645 ; Faraday, 'Experimental Researches,' 3266, 3267, 3268. 
to magnetic lines of force, refer, of course, to the two cases of similarly or oppositely directed lines, the positive direction of a line of force being reckoned as the direction in which a north-seeking pole on it would tend to move* ${ }^{*}$ The mutual coalescence or repulsion exerted between the lines of force of unlike or like magnetic poles is familiarly employed in the experimental illustration of magnetic phenomena, so well known since the researches of Professor Robison and Dr. Roget on the magnetic curves, and appears to have been recognized long beforef. It is believed that the present is the first distinct attempt to apply similar considerations to the illustration of electrodynamic relations between systems of conductors carrying currents, and between conductors of currents and magnet-poles.

The isodynamic lines, which are lines of magnetic force, tend to shorten themselves. A very hasty inspection of fig. 4 will show that if any one of the system of lemniscates were to "shorten itself," it would tend to bring the two centres nearer together. Consider each isodynamic line as a ring of some elastic material (as, for example, an indiarubber ring) stretched around a bundle of smooth wires, the cross section of the bundle having a perimeter corresponding in form to the isodynamic line under consideration. The maximum shortening of such an elastic ring would take place when the enclosed area was made a circle. In other words, the lemniscate-form isodynamics tend to become circles, and the two like parallel currents are mutually urged towards each other.

In figure 5 the shortening of the isodynamic lines, and their approach to the truly circular form, could only be accomplished by the wider separation of the two conductors from each other. Hence the mutual repulsion of two parallel conductors carrying oppositely directed currents.

Figures 6 and 7 show the lines of force above the parallel currents when these pass horizontally below the glass. In the case of like currents the lines coalesce. In the case of unlike currents they repel each other, and pass between the two wires in a direction vertical to the plane of the glass, where their characteristic form, as lines, is lost. The observation that the filings adherent to wires carrying like parallel currents are mutually attractive appears to have been first made by Davy.

Figure 8 illustrates the law of currents crossing one another at a point. In the two quadrants in which the currents both

* See Clerk-Maxwell, 'Electricity and Magnetism,' art. 489; L. Cumming, 'Theory of Electricity,' p. 19.1.

† See Musschenbroek, Dissertatio Physica Experimentalis de Magnete, crp. iv, exp, cxvii, and tab. iv. figs. $4 \& 5$, 
run to or from the central point, the lines of force tend to coalesce. In the alternate quadrants they mutually repel each other; and the angle of these quadrants tends to increase.

In figure 9 one carrent is carried horizontally below the glass, the other traverses the plane of the figure normally. The dissymmetry of the resultant distribution of the lines reveals the dissymmetrical nature of the force which tends to bring the currents into parallelism. Any shortening of the isodynamic lines would tend to move the vertical current from that quadrant over which the lines are unbroken. Figures have also been obtained with two currents crossing the plate in a vertical plane of incidence, but each at $45^{\circ}$ to the normal. With some distortion, these figures bear a general resemblance to figs. 4 and 5 in the two cases of the currents passing through the plate in similar or in opposed directions. In the former case their angular separation tends to diminish, in the latter to increase.

Figures 10,11, and 12 introduce the action of a vertical current upon a small magnetic needle lying on the glass plate. In the first case the needle lies in stable equilibrium almost tangentially to the isodynamic lines; in the third its position is reversed and unstable; in the second case it is set at right angles to the directive action of the current. The dissymmetrical action of the forces on its poles produces a couple tending to turn it about its centre, as would be inferred from an inspection of the lines of force of the figure.

Figures 13 and 14 illustrate a deduction from the theory of the magnetic shell. A conductor carrying a current is acted upon by a force urging it forward so as to make the number of like lines of force included within it a maximum*; that is to say, a north-seeking pole is attracted into a circuit on the side from which the positive current appears to circulate in a right-handed cyclical order (or in the same direction as the hands of a clock). Similarly the circuit is urged backwards from a contrary pole; and tends to make the number of unlike lines of force included within it a minimum. In the figures a current ascends through the plane of the figure on the left, and descends through it on the right, in a right-handed cyclical order as seen from the magnet. Hence a northseeking pole is attracted, and a south-seeking pole repelled.

Figure 15 results from the action of two magnet-poles upon a vertical conductor, which in this case is attracted between the poles.

Figure 16 illustrates the mutual tendency to rotation between a magnet-pole and a conductor carrying a current parallel to * Clerk-Maxwell, ' Electricity and Magnetism,' art. 490. 
the axis of the magnet. In the figure, where the vertical current passes upwards through the glass, the neighbouring southseeking pole (marked in position by a square dot) is urged round the current with a couple tending in a right-handed cyclical rotation. The couple is reversed, and acts in a lefthanded order, if either the current or the magnet-pole be reversed. The contrasted dissymmetry so produced is very curious, and the mutual displacement of the radial lines of force of the pole and of the circular lines of the current is very significant.

Figures 17 and 18 show lines of force arranged spirally in the field. In these a current passes upwards through the glass, while the pole of a magnet is placed vertically beneath : the current, in fact, passes through the magnet. The form of the lines of force is remarkable. No work would be done on or by an element of a vertical current in bringing it up to the centre along one of the spiral lines; for the work done by it in bringing it in the spiral path across the successive circular isodynamic lines of the current would be equal to that done upon it in carrying it across the successive radial lines of force of the magnet-pole. The equipotential surfaces of this field are consequently another series of spirals of an opposite cyclical order. In figure 17 the current running up through a south-seeking pole produced a right-handed spiral; in figure 18 , with a north-seeking pole the spiral is of the opposite order. Since the magnetic potential decreases from a magnet-pole with the inverse square of the distance, and since the inductive action of the current on a point in the plane of the figure also decreases according to the inverse square of the distance from the current, each branch of the spirals would be described by a moving point whose angular displacement from the arbitrary zero is simply proportional to the distance from the central point. The results of actual measurement of the spirals at successive distances of whole millimetres from the centre show as near an agreement with this supposition as the roughness of the method of procuring the curves permits. The number of branches of the spiral will clearly be proportional to the strength of the magnet pole. The obvious result of a "shortening" of the spiral lines would be to produce a rotational movement, such as we know to be produced on a free-magnet pole under the influence of a current traversing it longitudinally.

I am indebted to Mr. Robert Gillo, of Bridgwater, for the admirable photographic copies of the various figures.

June 19, 1878.

Phil. Mag. S. 5. Vol. 6. No. 38. Nov. 1878. 
Fhil.Mag. S 5.Vol.6. Pl.VI

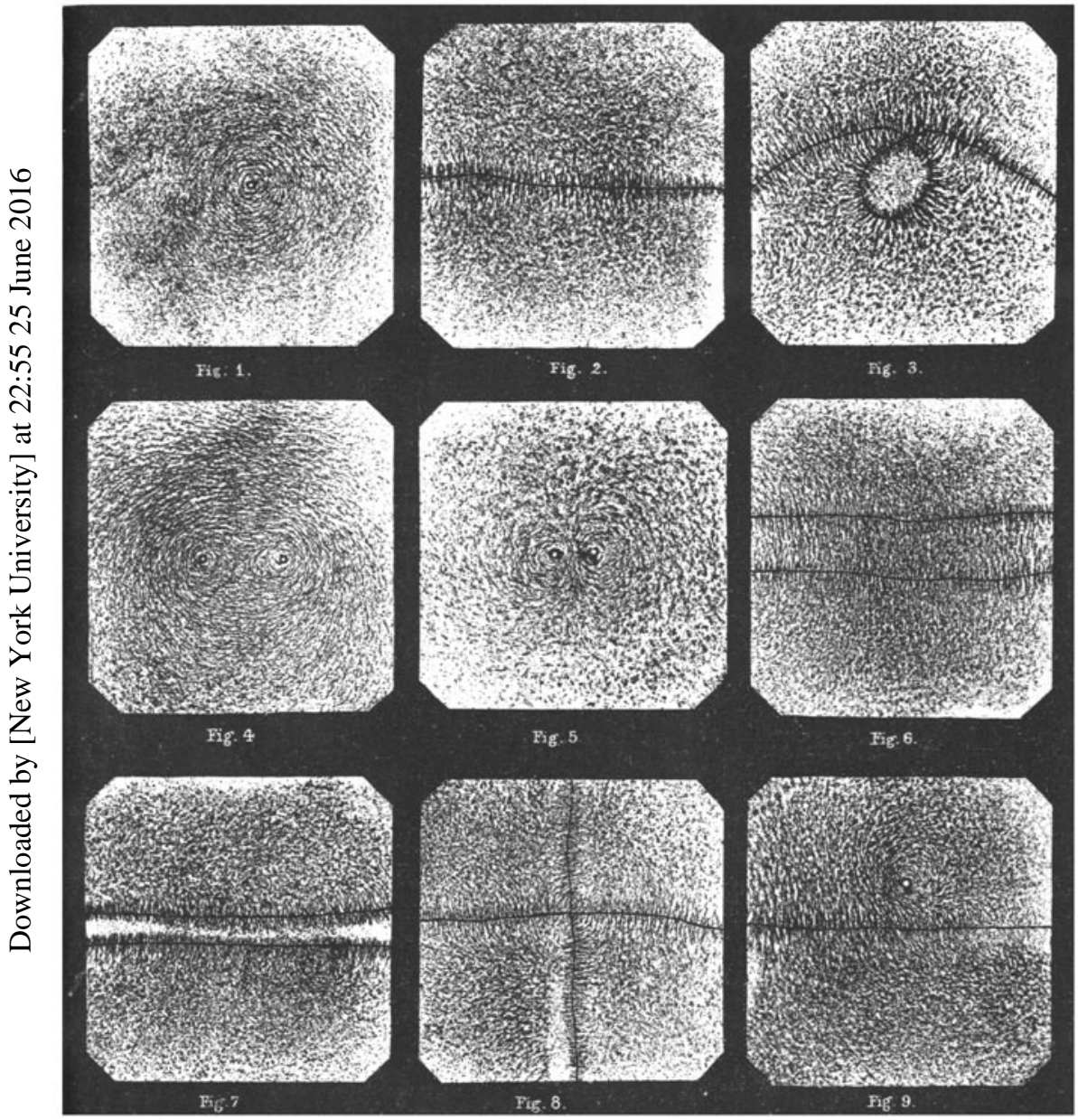

Whitem on Bast, Froto It tho to the Quzeen

\section{MACNETIC FIGURES}

ILLUSTRATING ELECTRO-DYNAMIC RELATIONS 
Phil Maǵ. S.5.Vol.6. Pl.VII

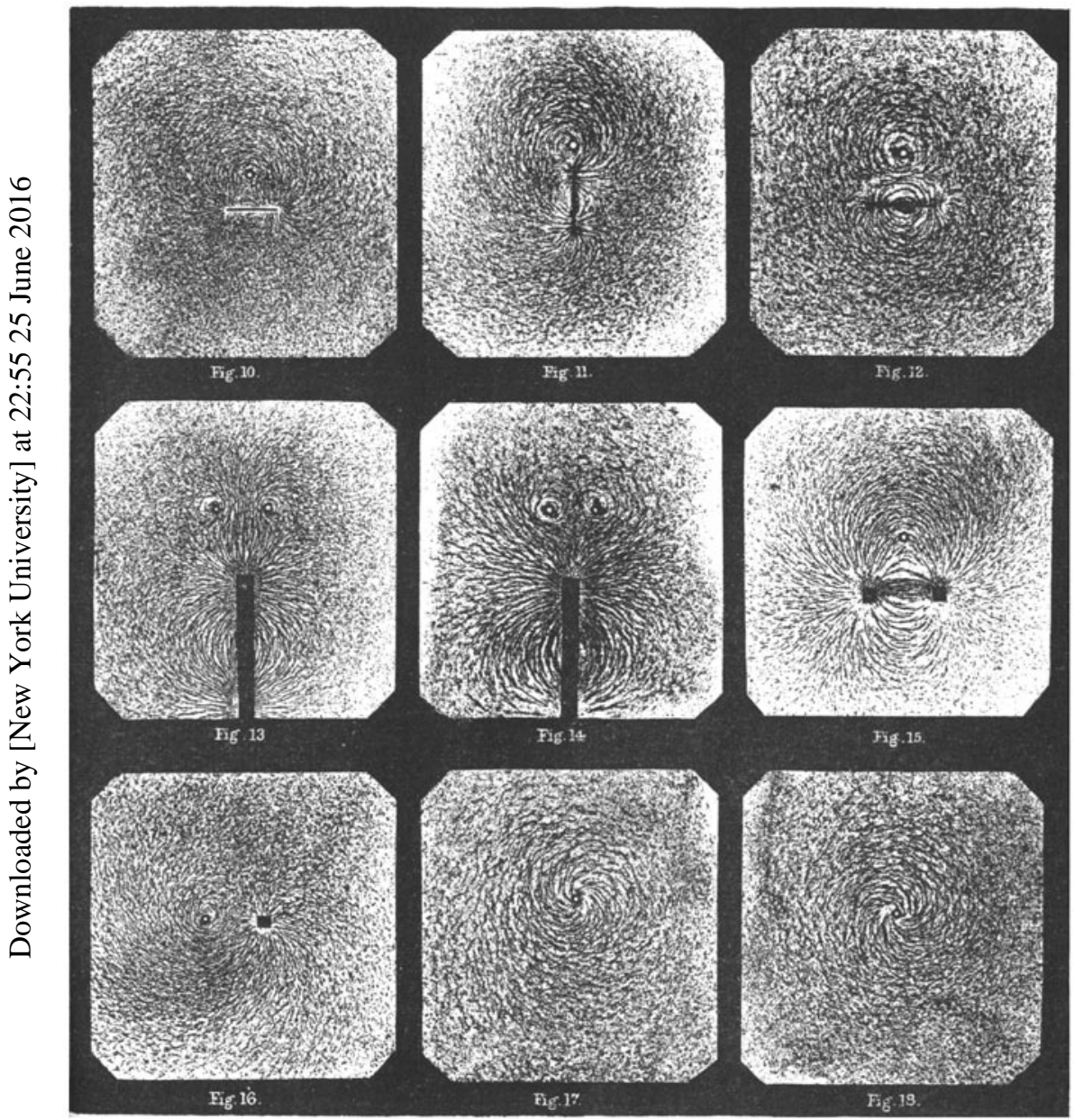

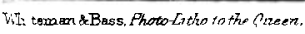

MAGNETIC FIGURES ILLUSTRATINC ELECTRO-DYNAMIC RELATIONS. 